\title{
SENSORY DISTURBANCES OCCURRING IN SCIATICA DUE TO INTERVERTEBRAL DISC PROTRUSIONS : SOME OBSERVATIONS ON THE FIFTH LUMBAR AND FIRST SACRAL DERMATOMES
}

BY

\author{
MURRAY A. FALCONER, GAVIN L. GLASGOW, and DAVID S. COLE \\ From the Departments of Neurosurgery and of Physiology, Otago University Medical School, \\ New Zealand \\ (RECEIVED 7TH OCTOBER, 1946)
}

IT is now widely accepted that many cases of severe and persistent sciatica are due to compression extrathecally of either the fifth lumbar nerve root by a protrusion from the fourth lumbar intervertebral disc, or the first sacral nerve root by a protrusion from the fifth lumbar disc (Bradford and Spurling, 1941 ; Falconer, 1944 ; etc.). It is also known that, in such cases, areas of sensory impairment, particularly of pain and tactile sensibility, often aippear in the affected leg. These areas, however, are difficult to chart, for the sensory loss is only partial, and it is generally assumed that they are confined to the foot and leg below the knee. In fact some authorities (Love and Walsh, 1938 ; O'Connell, 1943) presume that, if sensory changes extend above the knee, more than one nerve root must be involved; and that sacral roots within the theca are affected as well as the extrathecal nerve root at the level of the disc protrusion. Few appear to realize that, if sensation is tested carefully, a slight disturbance can usually be detected in the thigh and buttock as well as below the knee, even though only a single nerve root is involved, and that by careful study of these areas of sensory disturbance it is often possible to identify the exact nerve root concerned. Until a late stage in our own experience we did not realise that compression of either of these two nerve roots commonly produces patterns of sensory impairment which extend well above the knee. We then learnt that, if sensation is tested in a simple but standardized manner, it is frequently possible to outline a pattern of sensory change (verifiable by other examiners) which is characteristic of the particular nerve root, and hence is of diagnostic value. Since then further observations have been made which indicate that these areas are in reality dermatomes, and that the classical descriptions of the dermatomes by Head (1893), Déjerine (1914), and Foerster (1933), and even the more recent description by Keegan (1943) are faulty. Our observations instead support and expand ideas on these particular dermatomal areas put forward by Thorburn in 1893. We have also observed that areas of hyperalgesia may occur within the dermatomes, and further that, when pain is severe, sensory impairment may become more extensive than can be explained on a segmental basis alone.

\section{Method of Sensory Examination}

Patients suffering from sciatica, who wer admitted to the Neurosurgical Department of the Dunedin Hospital, New Zealand, were examine pre-operatively. Usually the symptoms had not been relieved by complete rest in bed for one month or longer, and were still sufficiently disabling to interfere with the patient's activities. Some had such severe pain as to be unable to rise from bed, but for the most part they were able to walk, although with discomfort. All cases were later proved at operation to be suffering from nerve root compression by a disc protrusion.

Like other observers, we found that sensory disturbances are detected more readily by painful than by other stimuli. Our method of examination was simple, and the only instrument required was a sharp needle or pin, or preferably a surgical needle mounted on a fine spring which, if compressed each time to the same degree, enabled stimuli of uniform intensity to be applied. The examination began with a series of pricks placed circumferentially a quarter to half an inch apart, first round $D$ the foot and then round the leg. The patient was prevented from watching what was going on. In this way the presence of any zone in which sensibility was not of quite as acute as elsewhere, or was.otherwise different, $N$ was determined. A test on the unaffected leg or on an $N$ upper limb acquainted the patient with the normal degree of pain produced by the standard stimulus. 


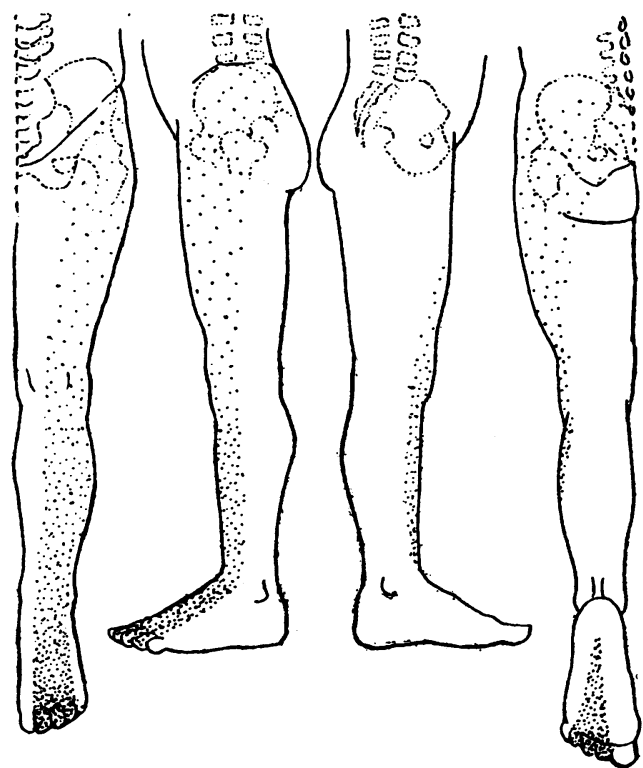

Fig. 1.-Pattern of area of hypoalgesia corresponding to the fifth lumbar dermatome.

Once a zone of hypoalgesia was discovered, all further testing was done from its interior outwards toward the normal skin, so that the boundaries might be determined. Tests had to be quick, with each series of pricks coming in rapid succession so that the patient did not tire. The patient signalled the moment he felt a change in the intensity of sensation, and the point at which this occurred was marked on the skin. When one boundary had been thus determined, the next was at once sought by applying the stimuli in a parallel or slightly radial direction half to one inch away. With practice the examiner could usually plot an area of sensory disturbance quickly. A good deal depended, however, upon the patient's perceptive ability, as the diminution in sensation was only slight, and not marked as in a peripheral nerve injury. The patient could usually distinguish readily between the blunt and sharp ends of a pin over the whole zone of hypoalgesia, and the only change he would notice was that at the boundary the prick of the pin suddenly became a little more vivid. We found that if the type of change to be expected was first explained to the patient the sensation could usually be tested successfully, but that if he were uncertain or apprehensive his replies would become misleading and even contradictory.

\section{Observations}

By employing this simple method of testing we noted three distinct types of sensory disturbance. Most often, a zone of hypoalgesia affecting a single dermatome extended along the lateral aspect of the limb from the foot to the buttock, its boundaries varying according to the nerve root involved. C

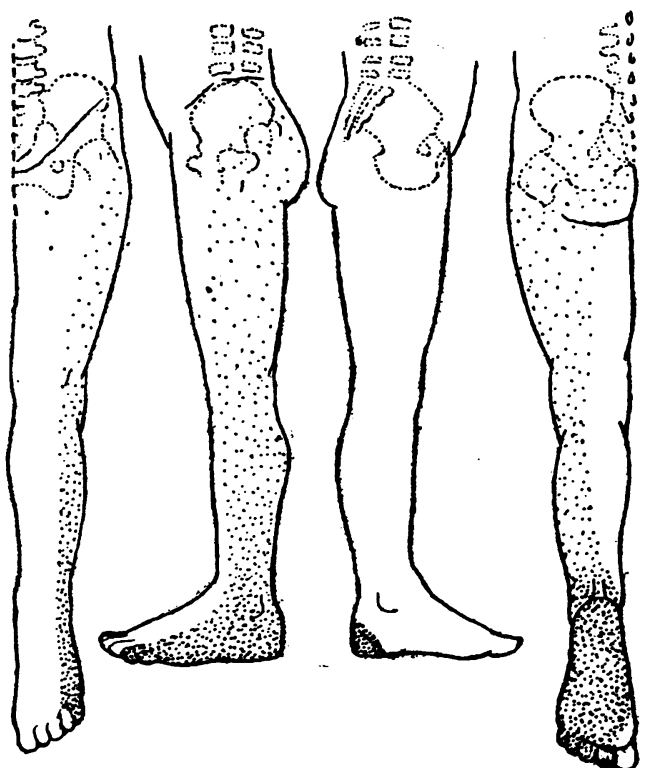

Fig. 2.-Pattern of area of hypoalgesia corresponding to the first sacral dermatome.

Sometimes, however, there were areas of hyperalgesia within the dermatome. And occasionally there was a condition of " spreading hypoalgesia" in which sensory impairment was much more extensive than could be accounted for on a dermatomal basis alone. Each set of observations will be described in turn.

Dermatome Hypoalgesia.-Hypoalgesic areas were detected in 33 out of 50 consecutive patients. These areas conformed to one or other of two main patterns and usually approximated to the full extent of the pattern, although sometimes they represented only its distal part. Each pattern was characteristic of a particular nerve root.

Fifth Lumbar Nerve Root Lesions.-This area involved a strip, from three to six inches wide, stretching from the foot to the lumbar region (Fig 1). At the foot it usually occupied the dorsum and the middle three toes, but sometimes it extended on either side to include the big and little toes, although it always spared the inner and outer borders of the foot. Inferiorly, it involved a wedge-shaped area of the sole. Above, it included the lateral surface of the leg, where its anterior boundary ran along the medial surface of the tibia. On the thigh it went in a gradual spiral on to the posterior surface, and then crossed the buttock to end in the lumbar region at the midline and at a variable level.

First SaCral Nerve Root Lesions.-This area resembled the fifth lumbar area in its general form, but it lay more laterally in the foot and leg and at a lower level in the lumbar region (Fig. 2). The two areas overlapped 
extensively. Distally, the first sacral area included the outer border of the foot and the little toe, but sometimes it extended on to the dorsum as far as the third toe. Inferiorly, the area sometimes included most of the sole and even the inner border of the heel. In the leg and thigh, the anterior boundary lay an inch or more lateral to that of the fifth lumbar area, while the posterior boundary was also further round the limb. The area ended above at the midline at a variable level in the lumbar region, a little below the level of the fifth lumbar area.

A prominent feature of both sensory areas was their extensive overlap (Fig. 4). This overlap was less in the foot, where it would seem that any particular area of skin is supplied by fibres from only two posterior nerve roots, than it is in the lumbar region, where there is overlapping distribution of fibres from three or more nerve roots. This explains why the boundaries of these hypoalgesic areas were most readily determined in the foot, and were much less distinct in the thigh and buttock. In several cases the detectable sensory loss was confined to a part of the usual area only, namely, to the foot and leg. This fluctuation in boundaries, particularly evident in the more proximal areas, was presumably due to variations in blocking of sensory impulses in the nerve root at the site of compression. When symptoms of sciatica were marked, the area of spontaneous hypoalgesia was usually large and approximated to

TABLE

\begin{tabular}{|c|c|c|}
\hline Type of disturbance & \multicolumn{2}{|c|}{ Number affected } \\
\hline $\begin{array}{l}\text { Dermatomal hypoalgesia } \\
\text { "Spreading hypoalgesia" } \\
\text { Hyperalgesia within a dermatome } \\
\text { No detectable sensory disturbance }\end{array}$ & \multicolumn{2}{|c|}{$\begin{array}{c}33 \\
3 \\
0^{*} \\
14\end{array}$} \\
\hline Total '.. & \multicolumn{2}{|c|}{50} \\
\hline $\begin{array}{l}\text { Subdivision of cases } \\
\text { showing dermatomal } \\
\text { hypoalgesia }\end{array}$ & $\begin{array}{l}\text { L } 5 \\
\text { derma- } \\
\text { tomes }\end{array}$ & $\begin{array}{l}\text { S } 1 \\
\text { derma- } \\
\text { tomes }\end{array}$ \\
\hline $\begin{array}{l}\text { Complete pattern } \\
\text { Incomplete pattern }\end{array}$ & $\begin{array}{l}9 \\
2\end{array}$ & $\begin{array}{r}17 \\
4\end{array}$ \\
\hline Total .. & \multicolumn{2}{|c|}{$\begin{array}{l}\text { Plus } 1 \text { case of com- } \\
\text { bined } L 5 \text { and } S 1 \\
\text { dermatomes }\end{array}$} \\
\hline
\end{tabular}

Analysis of sensory disturbances in 50 consecutive cases of sciatica, caused by lumbar intervertebral disc protrusions, later verified at operation.

* The three cases of hyperalgesia within the dermatome which are described in the text were collected before the present series of 50 consecutive cases was begun. the full extent of the dermatome, whereas when $\frac{\text { o }}{Z}$ symptoms were slight it was more restricted. There $\stackrel{\mathbb{D}}{ }$ were exceptions to this clinical rule, however, andō some patients with only slight pain exhibited extensive sensory impairment, whereas others witho moderate or even severe pain presented little if any. ${ }_{0}$ Presumably in patients with little or no sensory change relatively few sensory impulses were blocked in the compressed segment of the nerve root.

In each patient the boundaries of the sensory impairment were usually constant from day to day, and could be readily duplicated by other examinerso employing the same technique. The distributiono of sensory impairment as observed in the dorsume of the foot and toes, where sensory changes were $\mathbb{Q}$ most readily detected, gave a reliable clinicalo indication of which nerve root was affected, whereas higher up the boundaries were less certain. Sensory? changes on the sole was usually difficult to detect $\vec{\overrightarrow{ }}$ and delineate because of the thickness of the skin.W The value of careful charting of the sensory disturbances is shown by an analysis of our observations,? as given in the Table. In a series of 50 consecutive patients with sciatica due to intervertebral disơ protrusions, 11 showed hypoalgesic areas in the toes and dorsum of the foot conforming to theo first pattern (fifth lumbar dermatome), and $21^{\circ}$ showed areas conforming to the second pattern (first sacral dermatome). In all instances identity of the nerve root which was presumed to $\$$ involved was later confirmed at operation, theis showing the accuracy of the sensory testing. $\overline{3}$ one case the area of spontaneous hypoalgesia seemedo to involve the territories of both the fifth lumbar and the first sacral nerve roots, and at operation disco lesions were found at two different levels, eachs. compressing one of these roots. In addition, 3 patients showed "spreading hypoalgesia," while 14 had either no sensory disturbance, or changes so slight and inconstant that they could not be? charted.

Procaine Block.-In a few patients we succeeded in blocking with procaine solution the fifth lumbar? and first sacral nerve roots immediately lateral to the theca, producing areas of hypoalgesia, which presumably were of dermatomal character (a dermatome being defined as the area of skin supplied by a single posterior nerve root). These areas of induced hypoalgesia were essentially similar to those? occurring spontaneously in sciatica.

The method consisted of insertion into the theca in the midline of a fine-bore lumbar-puncture needle ats either the fourth or the fifth lumbar interspace. Using this needle as a guide to the depth and direction of the theca, a second needle was inserted parallel to it anck 
approximately half an inch to one side (Fig. 3). As the point of the second needle passed inwards, it could be felt to pierce the ligamentum flavum, and then, when about half an inch deeper than the guide, it came into contact with the nerve root just after it left the theca.

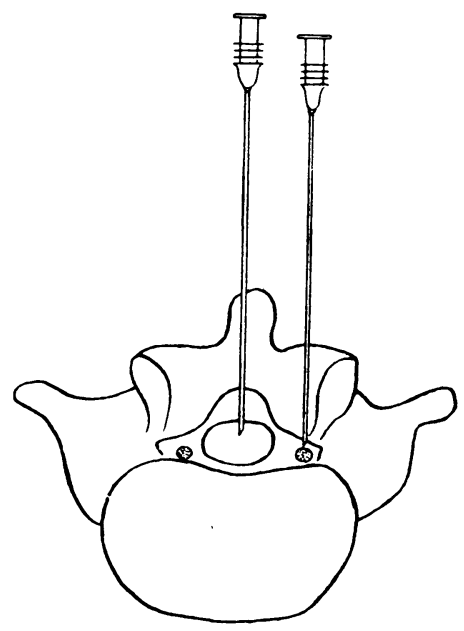

FIG. 3.-Position of needles placed for infiltration of an extrathecal nerve root with local anæsthetic solution. The central needle serves as guide. The lateral needle is employed for injection.

If the needle was correctly placed, movement of it caused severe pain referred to the leg, indicating that the nerve root was impinged upon, and if the stilette was removed no cerebrospinal fluid escaped. Once the needle was in position, $0.5 \mathrm{c} . \mathrm{cm}$. of $5 \%$ procaine solution containing a trace of adrenaline was injected, followed five minutes later by a second injection of the same amount. By employing procaine in small amounts of high concentration, its effects could be limited to the nerve root being tested.

On account of the difficulty in placing the needle correctly, especially in heavily-built persons, it was not always possible to make a successful injection. If the injection was technically successful, the patient usually remarked within a few minutes that his leg was becoming numb, while at the end of ten minutes, when the block was complete, there was a definite zone of hypoalgesia together with weakness of ankle movements and, in the case of the first sacral nerve root, absence of the ankle jerk. Injection at the fourth lumbar interspace blocked the fifth lumbar nerve, and injection at the fifth space blocked the first sacral. If the nerve root infiltrated was the one compressed, the patient was relieved of pain, and the straight-leg-raising movement was no longer limited. In fact these tests can be used to identify pre-operatively the affected nerve root, but, as they are difficult to perform, they are of academic rather than of practical value.

The areas of hypoalgesia produced by this rootinfiltration technique were almost identical with those occurring spontaneously in sciatica, the only points of difference being that the sensory impairment in the induced areas was usually a little more intense, and the boundaries, although similar in the foot, were a little wider apart proximally. These differences were small, and probably due to the local anæsthetic blocking all the sensory fibres in the root, whereas in compressive lesions some of the fibres usually escape.

By means of this infiltration technique we were able in some patients to produce nerve-root block in the sound leg, and to compare the induced sensory change with the spontaneous hypoalgesia existing in the affected leg. When the corresponding nerve root had been infiltrated, the two areas were essentially similar. By injecting the fifth lumbar nerve on one side, and the first sacral on the other in a case of low back pain without spontaneous sensory loss, we were able to induce a fifth lumbar dermatome hypoalgesia in one leg simultaneously with a first sacral dermatome hypoalgesia in the other, and so to control some of our other observations. This case exhibited clearly overlap of the two dermatomes (Fig. 4).

As a result of these observations with artificially produced nerve-root blocks, there can be little doubt that the areas which occur spontaneously in sciatica are in reality dermatomal areas.

Hyperalgesia within Dermatomal Areas.-In three patients an exaggerated response to the standard pain stimulus was observed within a dermatomal area. In two of these the area of hyperalgesia was confined to the buttock, below which it was continuous with a hypoalgesic area occupying the distal portion of the dermatome. In these two cases the increased reaction could be demonstrated only by pinprick, no change being found to tactile or thermal stimuli. In the third case the area of exaggerated pain response was larger, extending from the buttock as a narrow band down to the dorsum of the foot in the territory of the fifth lumbar nerve root. Within this area were smaller areas showing increased reaction to heat and to light touch. No definite change to cold stimulation was appreciated. Below the area of hyperalgesia there was a small patch of hypoalgesia along the outer part of the foot. An interesting and significant observation was made in this third patient after the fifth lumbar nerve root had been blocked extrathecally with procaine solution by our root-infiltration method. As the block developed, the increased reactions to pinprick, heat, and touch disappeared, and were replaced by a typical hypoalgesic dermatome. Later at operation, in this patient as in the other two, a disc protrusion was found compressing the anticipated nerve root, and was removed. We conclude from these observations that sometimes areas of hyperalgesia may develop within dermatomes, and that, when present, they tend to appear in the proximal part of the dermatome rather than in the distal. They may be 

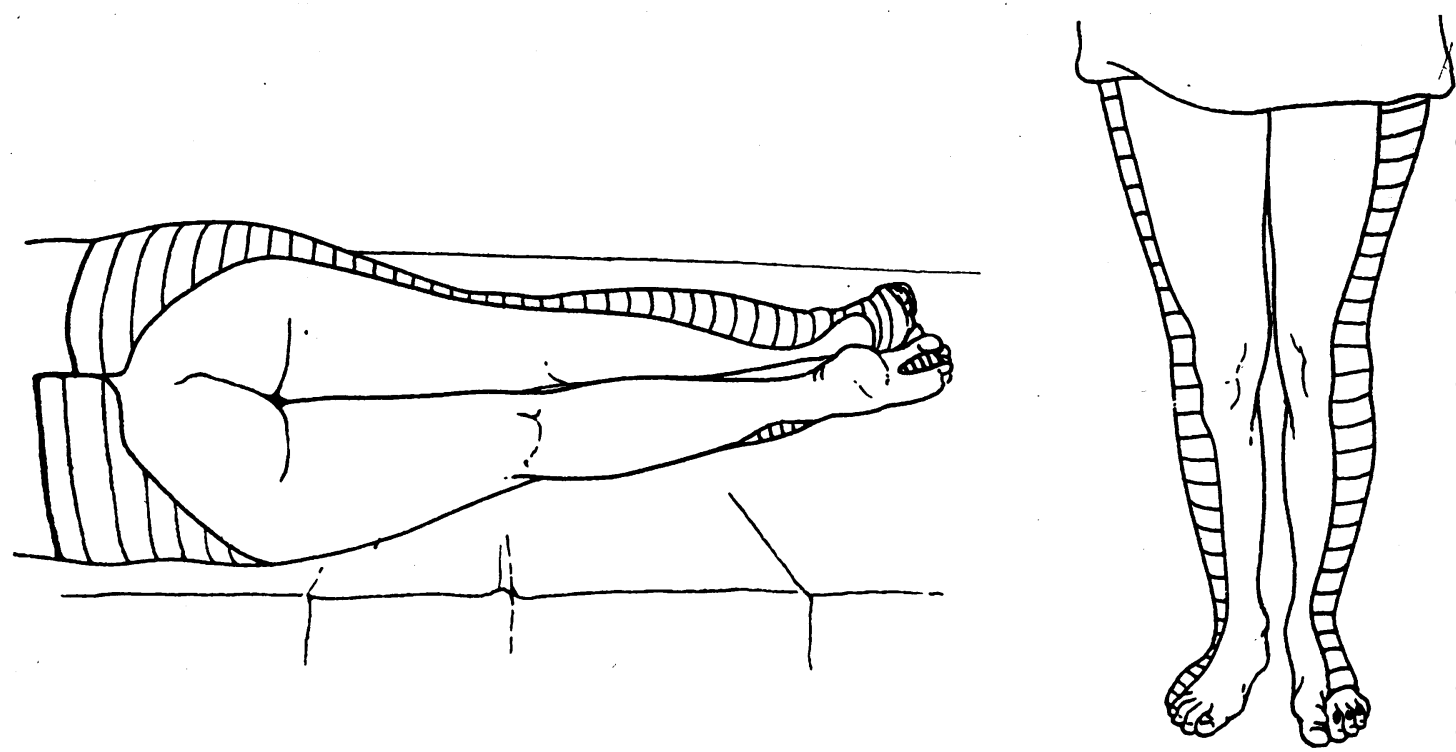

FIG. 4.-Extent of hypoalgesia produced by blocking with a local anæsthetic the fifth lumbar nerve root on the left side and the first sacral nerve root on the right side. Compare with Figs. 1 and 2. Note the degree of overlap.

associated with hypoalgesia in the distal part of the dermatome.

"Spreading Hypoalgesia."-Three patients, all confined to bed with sciatic pain, all had areas of hypoalgesia far more extensive than the distribution of a single nerve root. This extensive hypoalgesia was associated with marked weakness of all movements of the limb, and with gross limitation of straight-leg raising. In one case the hypoalgesia involved the whole hindquarter, its upper boundary fluctuating between the umbilicus and the costal margin (Fig. 5). In the other two, most of the lower limb below the groin was affected. Yet in all three at operation only a single disc protrusion was found compressing a single nerve root (the first sacral), and furthermore all symptoms and sensory signs completely disappeared after its removal.

In one case an observation was made which throws some light on the mechanism of " spreading hypoalgesia." The affected nerve root (first sacral) was infiltrated preoperatively with procaine solution according to our usual technique. This resulted in disappearance of the patient's pain for more than half an hour, and also of the limitation of straight-leg raising, while the motor power became nearly normal. While the procaine block was operating, a suspicion of a hypoalgesic zone encircling the lower abdomen persisted, but elsewhere in the lower limb the skin outside the boundaries of the first sacral root area now had normal sensibility. In other words, when the patient's pain was relieved, the superadded features of extensive motor weakness and of extensive sensory loss disappeared, leaving only those of a simple nerve-root block. The significance of this observation will be considered later.

\section{Discussion}

As a result of these various observations it seems evident that the sensory changes present in sciatio are usually due to compression of a single nerve rog $\overrightarrow{0}$ either the fifth lumbar or the first sacral. In sorm cases there are no detectable sensory signs, presumably because there is little or no interruption of sensory nerve impulses at the compressed segment of the nerve root. In the majority of patients however, areas of sensory impairment of a derma- $\bar{\alpha}$ tomal pattern are encountered. In a few instances areas of hyperalgesia may be met with, which usually involve the proximal portion of a dermatome and are often associated with hypoalgesia in the distal portion. In a further small group with severe spontaneous pain, the area of sensory loss may extend far beyond the anatomical confines of individual dermatomes.

Although current teaching based on Fœrsters' observations (1933) is that no sensory disturbance follows the interruption of a single posterior nerve root, this has not been our experience, nor that of Déjerine (1914). Fœrster, however, was apparently not concerned with the finer degrees of sensory impairment which we have charted, but rather with of areas of complete sensory loss. We agree with his observations that nowhere is cutaneous sensation abolished after interruption of only one posterior nerve root, but we have found that there is a slight (1)

\section{.}


diminution of its intensity within the segmental boundaries. As our dermatomal areas differ from those previously described, it seems appropriate at this stage to outline the classical descriptions of the dermatomes and the basis on which they were derived.
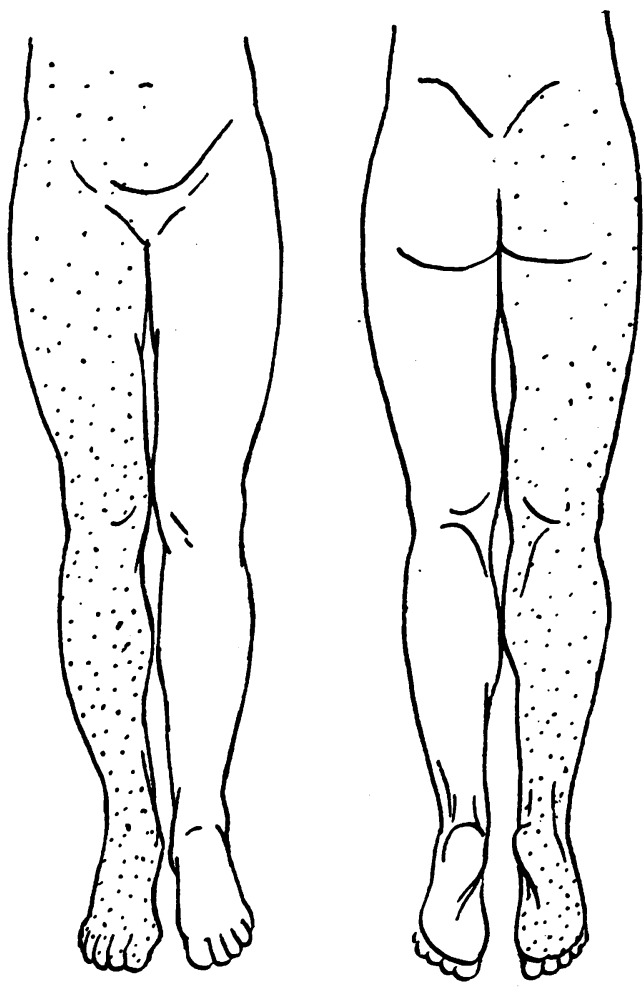

FIG. 5.-Extent of sensory impairment in a case of "spreading hypoalgesia" due to compression of the first sacral nerve root.

Classical Descriptions of Dermatomes.-The classical dermatome charts are based upon the work of Head (1893) and of Fœrster (1933) on man, and of Sherrington (1893) on the monkey. The boundaries of the fifth lumbar and first sacral dermatomes are similar in the schemes of Head and of Fœrster, but with one essential difference. Fœrster demonstrated extensive overlapping of his segmental areas, whereas Head found none, even on the trunk. In both schemes the fifth lumbar and first sacral dermatomes are confined to the leg and foot, and do not extend above the knee. In Fœrster's scheme (Fig. 6) the fifth lumbar dermatome occupies the whole anterior aspect of the leg, the dorsum of the foot, and also the medial part of the plantar surface of the foot. Head's boundaries (Fig. 7) are less extensive, for they do not include the toes or the sole of the foot. Fœrster described the first sacral dermatome as involving the greater part of the sole and the outer border of the foot, as well as an area on the posterolateral part of the leg. Head includes in addition the dorsum of all the toes. Fœrster arrived at his dermatomal areas by applying to man the method of residual sensibility employed by Sherrington in the monkey. As a therapeutic measure for various painful conditions, he divided a series of posterior nerve roots above and below a single posterior nerve root which he left intact. Subsequently he was able to chart between two areas of apparent analgesia an area of remaining sensibility, which he presumed to be a dermatomal area. By this method Fœrster in the course of operations upon many patients was able to outline what appeared to be the distribution of each posterior nerve root. At first sight this would seem to be a most accurate method of outlining the distribution of a nerve root, but reasons will be given later for believing that this is not so. Head outlined the distribution of skin lesions in cases of herpes zoster, and subsequently fixed the segmental level of his areas, by correlation with areas of sensory loss observed in cases of traumatic lesion of the spinal cord at known levels. His areas do not overlap, and it is interesting that Head himself did not claim them to be dermatomes. He was conversant with the early work of Sherrington, who had shown in the monkey that dermatomes as determined by the method of remaining sensibility do overlap. Head deduced that the areas he had mapped corresponded " to the supply, not of roots, but of segments of the spinal cord from which the roots in part arise." It has been left to subsequent writers to assume that Head regarded his areas as dermatomes.

Neither of these investigators made any attempt to correlate the arrangement of dermatomes in the limbs with that in the trunk. The result is an unconvincing, and apparently haphazard, arrangement of patches of skin with no underlying scheme. To bring the arrangement of dermatomes in the limb of the monkey into line with that in the trunk, Sherrington (1893) introduced the concept of anterior and posterior axial lines, regarded as "branches" of the mid-dorsal and mid-ventral lines entering the limb. On this basis he explained the fact that in the monkey, as in man, the roots in the centre of the plexus supplying a limb seemingly were not distributed to the proximal part of the limb. Déjerine (1914) rationalized the ideas and observations of other workers, and applied this concept of Sherrington to dermatomes in man. His chart is now widely used. When it is recognized 

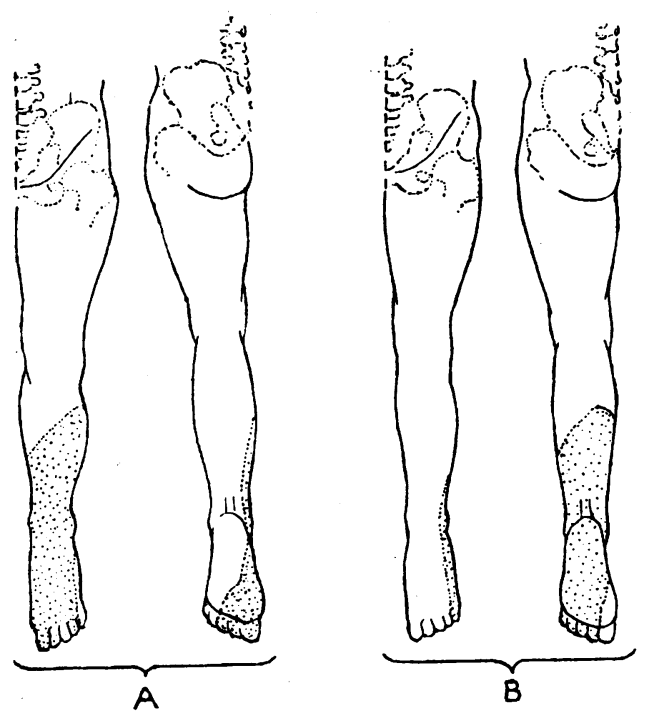

FIG. 6.-Extent of dermatomes, according to Fœrster : (a) fifth lumbar, and (b) first sacral.

however, that all limb dermatomes extend to the mid-dorsal line, the need for postulating Sherrington's axial lines disappears.

Thorburn (1893) in a critique of Head's paper described views on the sensory distribution of the various spinal nerves, which, as regards the fifth lumbar and first sacral nerves, are very similar to our own. It is a great pity that his paper has not been given the attention which it deserves. Thorburn studied areas of sensory loss in patients with disease or injury affecting known and limited regions of the spinal cord, or of isolated nerve roots, and he collected both personal observations and observations recorded in the literature. From these observations he deduced the segmental supply of various spinal nerves, by subtracting from the anæsthetic areas in his cases those areas whose nerve supply had already been established by anatomical dissection or otherwise. In this way he arrived at the areas of distribution of the remaining nerves. His method had obvious limitations, for it was dependent upon a sufficient supply of suitable clinical cases. By this means, however, he drew up a chart, which shows a distribution of the fifth lumbar and first sacral nerves that in many respects resembles our own (Fig. 8). He stressed that both these segmental areas, in addition to their distribution below the knee, extended upwards along the whole length of the thigh. He was unable to ascertain just how high they extended above this, and, largely on theoretical grounds, he depicted them in his chart as embracing the second sacral
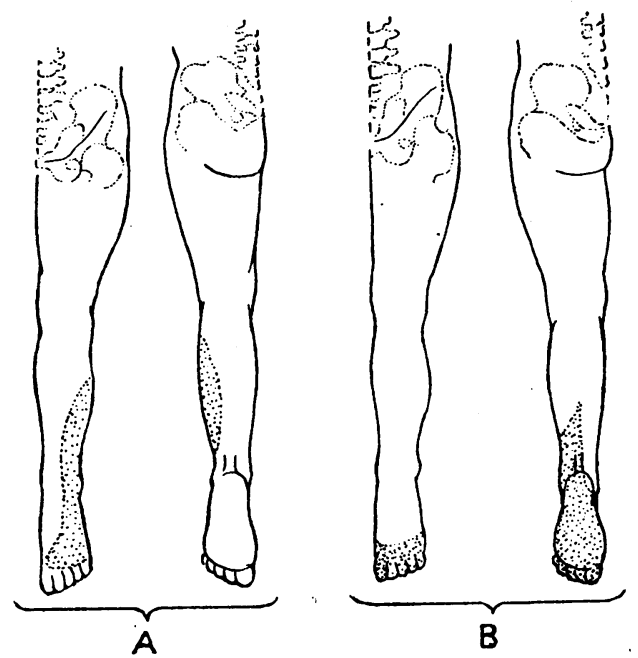

FIG. 7.-Segmental areas, according to Head : (a) fifth lumbar, and $(b)$ first sacral.

area on the back of the thigh. He also stressed in his text, though not so much in his diagram, that the $N$ various segmental areas overlap widely.

Recently Keegan (1943) has described areas of hypoalgesia in patients with lumbar intervertebra disc protrusions. He tested sensibility with pinprit by a method similar to ours, and his extensive case $\overrightarrow{0}$ material enabled him to define the distribution all nerve roots supplying the leg. His arrangement of the dermatome is simple and orderly, and analogous to the accepted arrangement in the trunk. The general configuration of his fifth and first sacral dermatomes (Fig. 9) is similar to those we have observed, but our findings differ in an important respect. There is no overlap in Keegan's charts, although he admits that the skin supply of each root may be greater than he shows.

The Anatomical Basis of Cutaneous Pain.-It has been well shown that the primary sensations of pain, touch, heat, and cold are each represented on the skin in punctate form (Walshe, 1942; Weddell, 1945). Underlying each spot, which is sensitive to touch, heat, or cold, are a group of specific structures, such as Meissner's corpuscles and Merkel's discs for touch, Ruffini's endings for heat, and Krause's end-bulbs for cold, each group of struc- $\frac{T}{0}$ tures being innervated by at least two different medullated nerve fibres. In the case of pain how- $N$ ever, there are no comparable structures, but instead plexuses of myelinated and unmyelinated fibres $\mathcal{N}$ situated in the dermis form the basis of the peripheral $\mathrm{\omega}$ receptor mechanism. These plexuses give rise to 

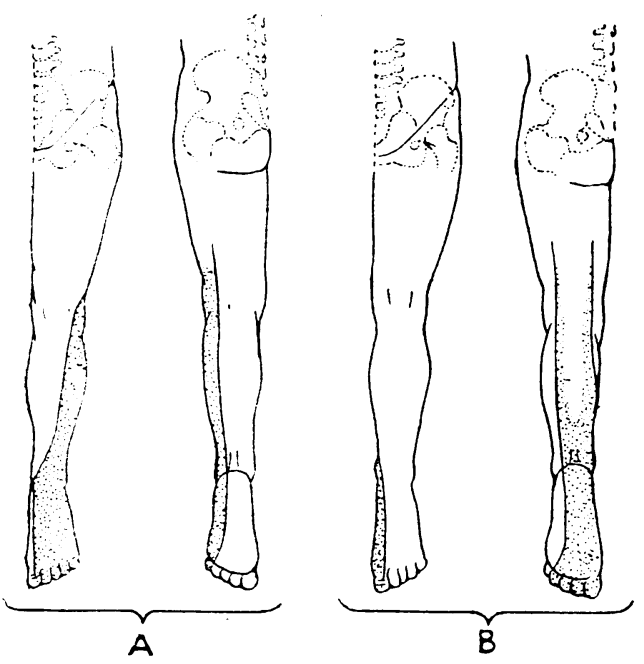

FIG. 8.-Extent of dermatomes, according to Thorburn : (a) fifth lumbar, and $(b)$ first sacral.

fine unmyelinated fibrils which end in beaded terminals that are situated in the dermis and the deeper layers of the epidermis. Within these plexuses it would seem that the branches from each parent fibre interlock with those from other fibres, but do not anastomose.

It is difficult to demonstrate a punctate distribution of pain in all parts of the body with a stimulus such as a pinprick, but Bishop (1944a), employing an electric spark of constant intensity as his stimulus, was able to show clearly that there are " high-spots" of sensitivity scattered over the skin, and that each is surrounded by an area of lower threshold 2 to 15 mm. diameter. By histological methods Bishop showed that each "high-spot" is supplied by branches from a number of fibres (Bishop, 1944b). $\mathrm{He}$ also found, when investigating the area of overlap of adjacent cutaneous nerves, that the response of a "high-spot" lying in the area of overlap may be lowered if one of the nerves supplying the area is blocked with a local anæsthetic. This indicates that the nerve fibres supplying a " highspot" may come from different cutaneous nerves, and also that the response of a "high-spot" to a given stimulus depends on the number of nerve fibres which are functioning.

If we postulate that the nerve fibres may come not only from separate cutaneous nerves but also ultimately from separate posterior nerve roots, dermatome hypoalgesia would be explicable by a diminution in the number of functioning nerve fibres supplying the pain " high-spots" within the territory of the affected posterior nerve root. Our
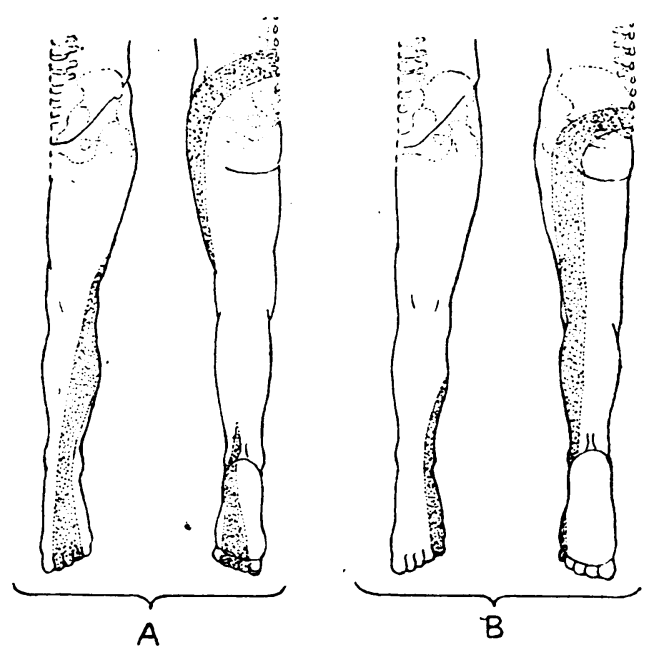

Fig. 9.-Extent of dermatomes, according to Keegan : (a) fifth lumbar, and (b) first sacral.

observations have shown that the overlap of the fifth lumbar and first sacral dermatomes is greatest proximally and least distally, and this can be correlated with the observation that sensory impairment is most vivid distally and least vivid proximally. Thus in the foot and leg, where each particular area of skin is innervated from approximately two posterior nerve roots, though not necessarily in equal proportions, a complete block of the nerve root affording the major supply will eliminate a high proportion of sensory fibres coming from the various " high-spots," and consequently will produce an appreciable degree of hypoalgesia. On the other hand in the thigh and buttock, where each particular area of skin is situated within the territory of three or more posterior nerve roots and presumably is supplied from each of them, blocking of one posterior nerve root will involve a much smaller proportion of the nerve fibres coming from the " high-spots," and so the resulting sensory impairment is less. Another factor contributing to the same result is that the component fibres of the fifth lumbar and first sacral nerve roots are probably distributed with a greater density in the distal parts of their dermatomes than in the proximal.

In contrast to the condition of dermatome hypoalgesia is the state of affairs existing when either the fifth lumbar or first sacral nerve root remains intact, while several above and below have been divided. This is the basis of the method employed by Sherrington and by Fœrster for determining the extent of the dermatomes. The number of nerve fibres which are left functioning after this procedure, and which 
produce an area of " remaining sensibility," is the same as that which, in patients with sciatica when only a single nerve root is blocked, produces a hypoalgesic dermatome. In this state of "remaining sensibility" the innervation of "high-spots" lying in the distal part of the distribution of the intact root is but little affected, since only a relatively small proportion of fibres are supplied by the adjacent severed roots. Consequently sensation in this distal part of the dermatome shows little if any impairment. In the thigh and buttock, however, where the major portion of the innervation comes from the overlapping nerve roots which are now severed, only a small number of nerve fibres remain. Consequently all " high-spots" have their innervation gravely impaired, with the result that it may be impossible to elicit sensation with a weak stimulus like a pinprick. Cobb (1919), for instance, found in patients with divided cutaneous nerves that near the margins of areas of sensory loss there are zones which are insensitive to a pinprick of $15 \mathrm{~g}$. weight, but which are sensitive to a pinprick of $30 \mathrm{~g}$. weight. These zones, which at first sight appear analgesic, are therefore in reality deeply hypoalgesic, and the areas in the thigh and buttock after isolation of either the fifth lumbar or first sacral nerve roots can probably be compared to them. These are the areas which we think represent the proximal portions of the dermatomes. Had Fœrster employed a massive stimulus, such as a hot disc of metal instead of a pinprick, he might have aroused sensation in these areas.

It is probable, then, that a pinprick may not be perceived in an area from which a large proportion, but not all, of the nerve supply has been removed. It has been shown that summation of the excitatory effects of a number of afferent nerve fibres is necessary before a reflex discharge is set up in an anterior horn cell (Eccles and Sherrington, 1930). Presumably the response of the secondary neurones in the pain pathways requires similar summation, and these neurones, like the anterior horn cells, have large numbers of terminations from different afferent nerve fibres in contact with them. Consequently, when a large proportion of the afferent nerve supply from any given area is out of commission, the few impulses which reach the spinal cord through the intact nerve fibres are unable to make the secondary neurones discharge. In addition it is probable that summation of many impulses is also necessary for the tertiary relay of the pain pathway. Granted then that impulses arising from stimulation of an area of little residual sensibility may not reach consciousness, we have still to explain why in the opposite circumstance removal of a comparable amount of innervation, $\bar{Z}$ as by blocking of a single posterior nerve root, $\stackrel{\mathbb{D}}{\leftarrow}$ produces a difference in sensibility which can be appreciated. The explanation is probably that, once the threshold of excitation in the secondary neurones is passed, they can signal by their fre- ${ }^{\circ}$ quency of discharge a small change in the intensity of stimulation coming from the posterior root fibres which by itself is less than the threshold necessary to cause a discharge.

Our hypothesis that single pain spots in the skin $\stackrel{\vec{s}}{+}$ may receive their innervation from more than one? posterior nerve root offers a ready explanation of $\frac{\overline{\bar{T}}}{\overline{2}}$ the sensory phenomena we have observed, both in sciatica and after blocking either the fifth lumbar or $\mathbb{Q}$ the first sacral nerves with a local anæsthetic. क However, some difficulty arises when we come to trace the peripheral nerve pathways by which fibres from the fifth lumbar and first sacral nerve roots $\vec{\omega}$ reach the various parts of their respective dermatomes. The fibres which supply the leg below the knee are undoubtedly conveyed by the great sciatic? nerve (fourth and fifth lumbar, and first, second, and $\vec{\circ}$ third sacral nerves) and its branches. The pathway? to the buttock is probably by way of the posterior N primary divisions of the spinal nerves. The 1938을 edition of Gray's Anatomy describes the posteriorprimary divisions of both the fifth lumbar and first 3 sacral nerves as possessing branches which help 8 supply the skin over the posterior aspect of the $\overrightarrow{0}$ buttock. The first sacral nerve is undoubted conveyed to the posterior aspect of the thigh by the small sciatic nerve (posterior femoral cutaneous? nerve-first, second, and third sacral). But it is difficult to see at first sight how the fifth lumbaro. nerve is conveyed to the thigh, because in the $\frac{\alpha}{\circ}$. conventional descriptions of British anatomical $\underset{\rightarrow}{\stackrel{0}{ }}$ textbooks the fifth lumbar nerve is excluded from $\overrightarrow{\overrightarrow{0}}$ the small sciatic nerve, and so from the cutaneous innervation of the thigh. The French anatomists, however, provide a way out of this dilemma, for they (Testut, 1930) describe the inferior gluteal nerve (fifth lumbar, and first and second sacral)? and the small sciatic nerve (first, second, and third $\frac{5}{3}$ sacral), not as two separate nerves as their British confrères do, but as a single nerve which gives motor branches to the gluteus maximus muscle and sensory branches to the posterior aspect of the thigh and $\frac{}{1}$ buttock.

Finally, we have to account for the marked overlap of distribution of the fibres of neighbouring nerve $N$ roots once they reach the skin. Our observations have shown clearly that, in the leg, the fifth lumbar and first sacral dermatomes occupy broad zones, $\omega$ each covering a large part of the circumference of 
the limb. Similarly, the dermatomes of the trunk are probably also broad, for we have observed a zone of hypoalgesia from three to three and a half inches wide, encircling one side of the chest, after division of only a single posterior nerve root (the fourth thoracic). In considering why the dermatomes are so broad, the arguments of Tower (1943) bearing upon the area of distribution of a single pain fibre should be remembered. She has pointed out that the spreading cutaneous hyperalgesia of Lewis, which is seen after pinching, faradizing, or otherwise injuring a small area of skin, would be explicable without the necessity of assuming a special nocifensor system of innervation, if a single pain fibre were distributed over a much larger area than most authorities have hitherto believed possible. She is prepared to postulate unit areas in the skin of the human forearm measuring nine to twelve $\mathrm{cm}$. long by three to four wide. Her concepts have yet to be confirmed or refuted by other methods, but, if they are true, they will help considerably in the understanding of the mechanisms of overlap between two dermatomes.

Fœrster stimulated at operation the distal ends of various posterior nerve roots which he had divided, in an attempt to mark out segmental areas by observing the extent of the resultant cutaneous vasodilatation (the "antidromic" response of Stricker and Bayliss). Lewis (1942) quotes him as noting that this caused a burning sensation to appear in the area of overlap of other roots. The conditions under which Fœrster made his observations are paralleled by those seen in nerve-root compression. In this latter condition the conduction of impulses through the affected root can be assumed to be blocked, while impulses probably arise at the damaged region and pass centrifugally down the nerve to the periphery. According to Lewis' hypothesis, the centrifugal impulses on reaching the terminations of the pain fibres cause liberation in the skin of a pain-producing substance (P substance), which lowers the threshold of the pain endings of the overlapping roots and produces hyperalgesia. It is significant for this explanation that in each of our three cases the hyperalgesia appeared in the proximal regions, where overlap from the neighbouring nerve roots is greatest.

The phenomenon of " spreading hyperalgesia" cannot be explained on known anatomical grounds, for the areas it involves extend both far above as well as far below the segmental level of the affected nerve root. In each of our three cases, "spreading hypoalgesia " was associated with pain of marked severity. Another noticeable feature was widespread motor weakness. It is significant that, after pain had been relieved by operative removal of a single nerve-root compression, the "spreading hypoalgesia," as well as the widespread motor weakness, disappeared in all three cases. It is also significant that both these features disappeared temporarily in our third case, when pain was relieved by a procaine block of the affected nerve root. These observations suggest that the severe pain, itself, is the factor responsible for " spreading hypoalgesia." Ray and Wolff (1945) have shown clearly that with pain of great severity the afferent impulses may spread widely within the spinal cord, thus affecting neurones with which they are not normally in functional communication. Their evidence is that in a patient with a unilateral loss of pain sensibility due to an anterolateral chordotomy, the application of a severe nocuous stimulus to the analgesic limb causes the patient to feel pain at the comparable point in his opposite sound limb. We have in part confirmed their observations, and the only likely explanation is that the intense bombardment by the incoming pain impulses excites widespread discharges of internuncial neurones, and hence eventually of the secondary neurones of the pain pathway for the opposite limb. The impulses from these secondary neurones, on being transmitted up to the sensorium, are then interpreted as arising in the sound limb. If the excited internuncial neurones can give widespread inhibitory effects as well as excitatory, such inhibition of the secondary neurones relaying impulses from adjacent roots would account for " spreading hypoalgesia."

Dermatomes in a Case of Cauda Equina Injury.-It would seem that the chief reason why our observations on the fifth lumbar and first sacral dermatomes differ from those of Fœrster is that we employed a different criterion for sensory testing. Fœrster, like ourselves, used light pinprick, but he marked out areas of apparent analgesia, taking as his boundary the line at which an area without sensation changes to one of pain perception. We, however, mapped out areas of hypoalgesia, taking as our boundary the line of change between slightly impaired sensation and completely normal sensibility. Consequently our dermatomal areas are more extensive than his; and moreover we have been able to delineate areas of sensory impairment after a lesion of only a single nerve root, which would not have been possible had we adopted Fœrster's criterion.

We were fortunate in being able in a case of gunshot wound of the cauda equina to compare our method of sensory testing with that of Fœrster, and incidentally to substantiate the validity of many of the arguments we 
have raised in the discussion. This patient, an unimaginative middle-aged man, had suffered a complete interruption of the fifth lumbar and all the sacral nerve roots on one side of his body. We were thus able to study the overlap between the fourth and fifth lumbar dermatomes. His injury had been caused sixteen months earlier by an 0.22 rifle bullet, which had penetrated his back in the midline between the third and fourth lumbar vertebræ, and had transversed the theca to lodge in the centre of the third lumbar intervertebral disc. The cauda equina had been explored a few hours sufficiently to cause a painful sensation without blistering on momentary application to the intact skin, the extent of the complete sensory loss was found to be much smaller (depicted in shade). It consisted of two areas, one in the buttock and the upper part of the thigh, and one in the calf, sole, and outer half of the dorsum of the foot, with an isthmus in between. Two similar areas of complete sensory loss were also found when sensation was tested with a heavy pinprick sufficient to draw blood. It was noteworthy that the lateral boundary of the upper area of complete analgesia lay from one and a half to two

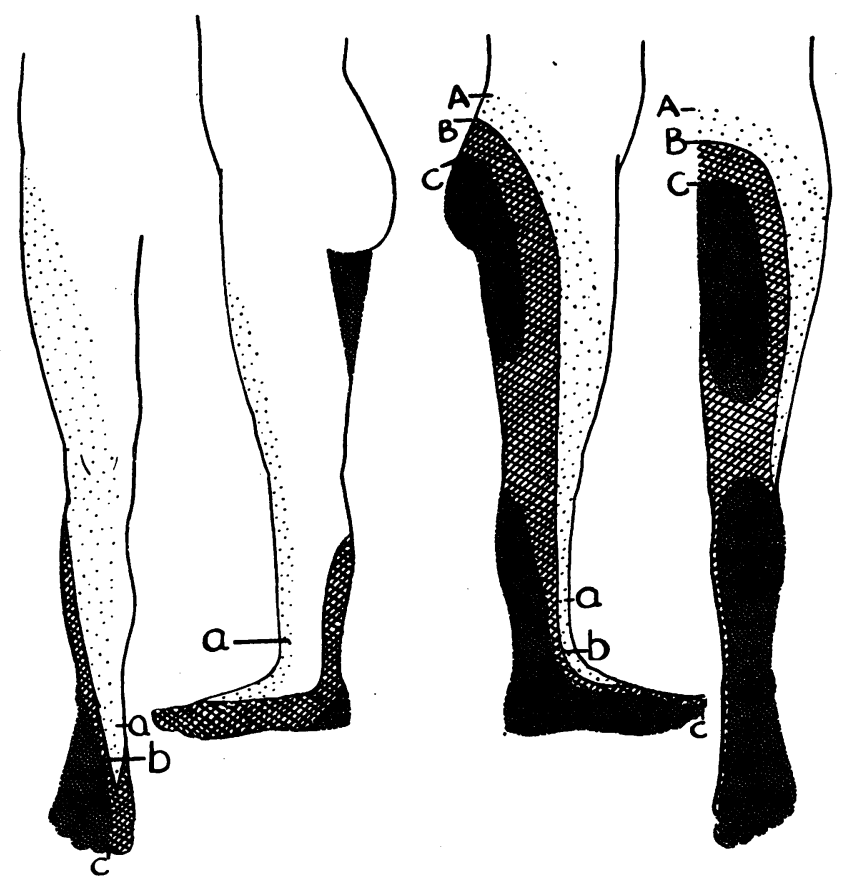

Fig. 10.-Diagram showing extent of sensory disturbance after section of fifth lumbar and all sacral nerve roots. Crosshatching shows the area of loss of sensation to light pinprick. In the shaded area there was complete loss of sensation to massive pain stimuli. In the dotted area there was hypoalgesia.

after injury, and the roots of its left half were found to be intact, whereas those of its right half with the exception of the outermost one (presumably the fourth lumbar root) were severed.

When this man was tested with painful stimuli, three sensory levels could be made out (Fig. 10). When tested with light pinprick, an area of seemingly complete sensory loss (depicted by cross-hatching in Fig. 10) was found extending down the whole of the posterior aspect of the limb from the buttock in the midline above to the toes below. It embraced the outer two-thirds of the dorsum of the foot as well as the sole and inner border. When sensation was then retested with a disc of metal, heated inches within the corresponding boundary for sensory loss to light pinprick, whereas the medial boundaries of the two areas corresponded closely. In the calf and foot the lateral and medial boundaries of the lower area of complete analgesia approximated to within half an inch of those of the area of sensory loss to light pinprick.

Next, sensation was tested with light pinprick in the same manner as we would have employed in a case of $\bar{N}$ sciatica. In this particular patient we found that above $?$ and anterior to the area of sensory loss was an area of $\mathrm{N}$ slight hypoalgesia. (depicted by dotting). Its upper $N$ boundary ran along the iliac crest, wound round the front of the thigh, and then passed down the anteromedial 
aspect of the leg towards the base of the great toe. The difference in feeling between this zone of hypoalgesia and the normal skin was very slight, but each time the testing needle crossed its boundaries the patient winced. We found no comparable zone of hypoalgesia on the medial side of the areas of analgesia.

This case therefore demonstrates clearly how, with painful stimuli of differing severity employed with differing criteria of sensory change, differing sensory levels can be made out. The area of sensory loss to light pinprick (depicted by cross hatching) corresponds closely to the territories of the fifth lumbar and all sacral nerve roots as described by Head. The demonstration that this area was really compounded of zones of deep hypoalgesia as well as of true analgesia supports our contention that, had Fœrster employed a more massive pain stimulus when investigating his areas of " remaining sensibility," he would have found that the fifth lumbar and first sacral nerve roots have a cutaneous representation in the thigh and buttock. Finally the zone of hypoalgesia above and anterior to the area of sensory loss corresponds exactly to the upper and anterior part of the fifth lumbar dermatome as observed by us in sciatica.

This case also demonstrates clearly how greatly the dermatomes overlap, and how this overlap is greatest in the thigh and buttock and is least in the leg and foot. As the fifth lumbar and all sacral roots had been severed, the lowest nerve root still subserving sensation was the fourth lumbar root. On reference to Fig 10, it can be seen that the boundary A-a of the zone of hypoalgesia represents the upper detectable limits of the fifth lumbar dermatome, for reasons discussed in the preceding paragraph; the boundary B-b of the area of sensory loss corresponds to the lower limit of the fourth lumbar territory, as Fœrster would have detected it by light pinprick ; whereas the boundary $\mathrm{C}-\mathrm{c}$ of the areas of true analgesia approximates more closely to the true lower limits of the fourth lumbar dermatome. The overlap of the fourth and fifth lumbar dermatomes would, therefore, seem to extend over at least three to four inches in the buttocks (from the points $\mathrm{A}$ to $\mathrm{C}$ ), and only over an inch or less in the lower leg (from the points a to c). Thus, the various observations in this case of cauda equina injury substantiate most of the major premises which were raised in the discussion of dermatome hypoalgesia.

\section{Conclusions}

We therefore conclude that the various sensory disturbances, which occur in sciatica, originate in changes affecting a single dermatome, and can be explained by the hypothesis that each particular area of skin is innervated from two or more posterior nerve roots. These disturbances are seldom marked, and can only be detected by careful sensory testing. They result from lesions of either the fifth lumbar or the first sacral nerve roots, and conform to a pattern which is characteristic of the particular nerve root, and hence is of diagnostic value.

Our observation that a lesion of either of these nerve roots commonly produces a sensory disturbance, not only in the leg and foot, but also in the thigh and buttock, is of great importance. It casts doubt on the accuracy of the classical descriptions of the dermatomes and on the validity of the methods by which they were determined. From the clinical point of view, the disclosure of sensory impairment in the thigh and buttock in a patient with sciatica can no longer be necessarily regarded as evidence that several roots of the cauda equina are being involved, or that the patient is hysterical because his sensory pattern does not conform to the classical dermatomal charts, explanations which have both been often considered by clinicians in the past. It is our experience that if, in a patient with sciatica, a sensory disturbance-even though slight-can be demonstrated, careful plotting of its boundariesespecially on the dorsum of the foot, where the sensory changes are most readily detected-will usually afford a reliable indication as to the identity of the affected nerve root.

In this paper we have confined our observations to two dermatomes, the fifth lumbar and the first sacral, and have shown that they have a simple and orderly distribution extending the whole length of the lower limb, and one which is analogous to the accepted distribution of the dermatomes in the trunk. It seems unlikely that a similar arrangement should not occur with the other dermatomes of the limbs, both upper and lower. Clinical material exhibiting lesions suitable for the study of these dermatomes is sparse, but we have seen two cases of compression of the seventh cervical nerve root by an intervertebral disc protrusion, which exhibited sensory impairment extending the length of the upper limb from the fingers to the mid-dorsal region.

We wish to thank the Medical Research Council of New Zealand for defraying the expenses incidental to this investigation; also Prof. J. C. Eccles, Head of the Department of Physiology, for his help and encouragement throughout the investigation; and Prof. W. E. Adams, Prof. F. H. Smirk, and Dr. Murray McGeorge for advice and help with this paper. We are indebted to Miss Valerie Tregonning for the diagrams and Miss Evelyn Madigan for technical assistance. 


\section{REFERENCES}

Bishop, G. H. (1944a). J. Neurophysiol., 7, 71.

- (1944b). Ibid, 7, 185.

Bradford, F. K., and Spurling, R. G. (1941). "The Intervertebral Disc." Springfield, Illinois.

Cobb, S. (1919). Arch. Neurol. Psychiat. Chicago, 2, 505.

Déjerine, J. (1914). "Sémiologie des Affections du Système Nerveux." "Paris.

Eccles, J. C., and Sherrington, C. S. (1930). J. Physiol., 69, 1.

Falconer, M. A. (1944). N. Zealand med. J., 43, 58.

Fœrster, O. (1933). Brain, 56, 1.

Head, H. (1893). Ibid., 16, 1 .

Keegan, J. J. (1943). Arch. Neurol. Psychiat. Chicago, 50,67 .
Lewis, Sir T. (1942). “Pain.” New York.

Love, J. G., and Walsh, M. N. (1938). J. Amer. med. Ass., 111, 396.

O'Connell, J. E. A. (1943). Brit. J. Surg., 30, 315.

Ray, B. S., and Wolff, H. G. (1945). Arch. Neurol. Psychiat. Chicago, 53, 257.

Sherrington, C. S. (1893). Philos. Trans., 184, B, 641. D Testut, L. (1930). "Traité d'Anatomie Humaine." Paris.

Thorburn, W. (1893). Brain, 16, 355.

Tower, S. S. (1943). Proc. Ass. Res. nerv. ment. Dis., 23, 16.

Walshe, F. M. R. (1942). Brain, 65, 48.

Weddell, G. (1945). Brit. med. Bull., 3, 167. 